\title{
Comparative Aspects in Control Strategies for Hybrid DC/DC Converters
}

\author{
Herminio Martínez-García and Jordi Cosp-Vilella \\ Barcelona College of Industrial Engineering (EUETIB) \\ Department of Electronics Engineering \\ Technical University of Catalonia (UPC). BarcelonaTech \\ Campus de la Escuela Industrial de Barcelona. C/ Comte d'Urgell, nº 187 \\ 08036 - Barcelona. SPAIN \\ Phone Number: +34.93.413.72.90; Fax Number: +34.93.413.74.01 \\ E-mail: herminio.martinez@upc.edu,jordi.cosp@upc.edu
}

\begin{abstract}
Linear-switching hybrid DC/DC converters consist of a voltage linear regulator (classic NPN or nMOS topologies and LDO) connected in parallel with a switching DC/DC converter. In order to control these hybrid structures, different strategies exist, allowing to fix the switching frequency as a function of some parameters of the linear regulator. This article compares two control strategies that, although they can be applied to the same circuital structure of linear-assisted converter, they are slightly different. The first one, reported in previous literature, cancels completely the average current through the linear regulator in steady state to achieve a reduction of power losses. Thus, the efficiency of the whole system increases and almost equals the one of the standalone switching converter. The proposed approach, in spite of a slightly increment of linear regulator's losses, reduces the output ripple that is caused by the crossover distortion of linear regulator output stage.
\end{abstract}

\section{Key words}

DC-DC Switching Converters, Voltage Linear Regulators, Linear-Assisted DC-DC Voltage Regulators.

\section{Introduction}

The present article compares two control strategies that can be applied to the same circuital structure of a linearassisted converter. However, they are significantly different. The first one (that we will denominate ' $A$ ' and is reported in previous literature [1],[2]), tries to completely cancel the current through the linear regulator in the steady state in order to achieve a reduction of power losses. Thus, the efficiency of the whole system increases and almost equals the one of the switching converter. It considers as the "main" block the switching DC/DC converter, and the linear regulator as an auxiliary module.

On the other hand, this proposal (strategy ' $B$ ') allows some average current flow through the linear regulator. In spite of a slightly increase of linear regulator's losses, this strategy reduces the output ripple due to the crossover distortion of its output stage. Thus, this approach considers as the "main" block the linear one, and the switching one as the auxiliary module.

\section{Control Strategy ' $A$ '}

The first of the two strategies of control that is considered in the current article is implemented on the converter of figure 1, where the implementation of the linear-assisted converter consists of a linear regulator (including transistors $Q_{2 a}$ and $Q_{2 b}$, which form an output complementary push-pull stage) and a switching DC/DC converter connected in parallel with the first one. In this case, the switching converter is a step-down type (buck converter) without the output capacitance. With this strategy, the switching converter is considered as a "main" block, whereas the linear regulator is considered as the auxiliary block that "assists" the first one when it is not able to provide output currents with high variations (that is to say, with high slew rate of the load current).

The control strategy consists of sensing the current through the linear regulator and, transforming it into a voltage (thanks to the current sensing element $R_{m}$ ), controlling the switching frequency of the DC/DC switching converter. The main objective of this one is to provide all the load current in steady-state conditions (to obtain high efficiency of the whole system). Thus, in steady state the linear regulator does not provide current to the load, although it maintains the output voltage to an acceptable DC value.

However, when variable output loads are driven, the linear regulator provides high transitory changes of the current in order to maintain constant the output voltage of the whole structure (figure 2). Therefore, we can name to this type of control as strategy control with null average linear regulator current. Resistors $R_{1}$ and $R_{2}$ of the Schmitt trigger determine the width of its hysteresis cycle 
and, thus, the maximum value of the switching frequency of the $\mathrm{DC} / \mathrm{DC}$ converter.

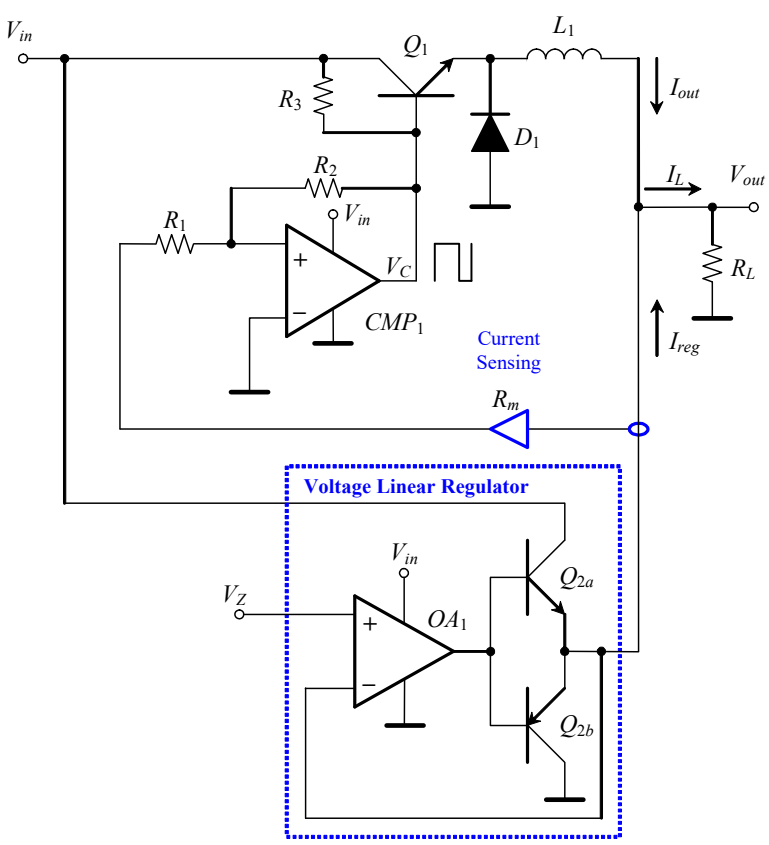

Fig. 1. Basic structure of linear-assisted converter with control strategy ' $A$ '.

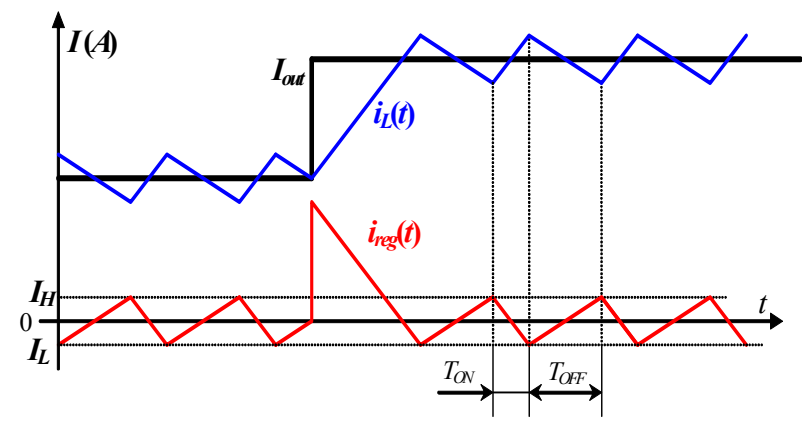

Fig. 2. Principle of operation of a linear-assisted converter with control strategy ' $A$ '.

This control strategy allows to obtain an efficiency almost equal to the switching converter, because the current by the linear block and, thus, the dissipation in its pass transistors is, practically, neglected. Nevertheless, it suffers from a limitation referring to maintaining the output voltage free of significant ripples due to the wellknown crossover distortion inherently present in push-pull amplifiers.

Notice that although the output voltage remains, in average, around the output voltage prefixed by means of the reference $V_{Z}$, the ripple is approximately $0.6 \mathrm{~V}$ (figure 3 ). The reason of this ripple is due to the output complementary transistors in the linear regulator. In fact, in every switching period, they switch from their cut off region to their linear zone and vice versa. As a consequence, during the transitions from cut off to conduction and vice versa, due to the well-known crossover distortion, a lack of regulation of the output voltage takes place, originating a ripple that, in certain applications, can not be tolerated.

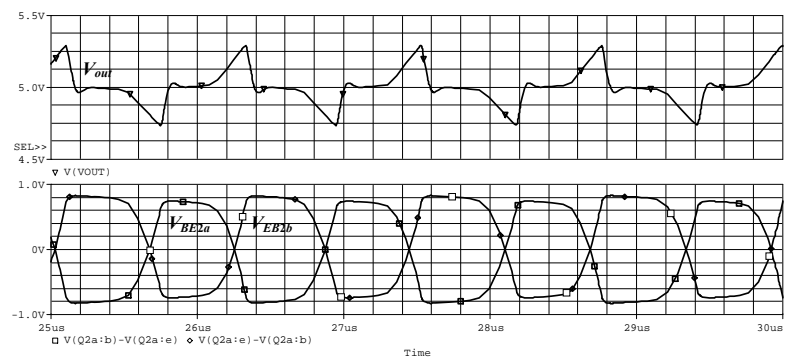

Fig. 3. Detail of the output voltage ripple and base-emitter voltage of transistors $Q_{2 a}$ and $Q_{2 b}$ of the linear regulator in the steady state for the linear-assisted converter in figure 1 .

\section{Control Strategy ' $B$ '}

The proposed strategy in this paper is analyzed using the step-down switching converter shown in figure 4 [3], [4]. The linear regulator consists of a push-pull output stage (transistors $Q_{2 a}$ and $Q_{2 b}$ ). In this strategy, the main objective of the DC-DC switching converter is to provide most of the load current in steady-state conditions to obtain also a good efficiency of the whole system. Thus, thanks to the incorporation of the reference voltage $V_{\text {ref }}$ at the inverting input of the analog comparator, the linear regulator provides a small part of the load current in steady state, maintaining the output voltage to an acceptable constant value.

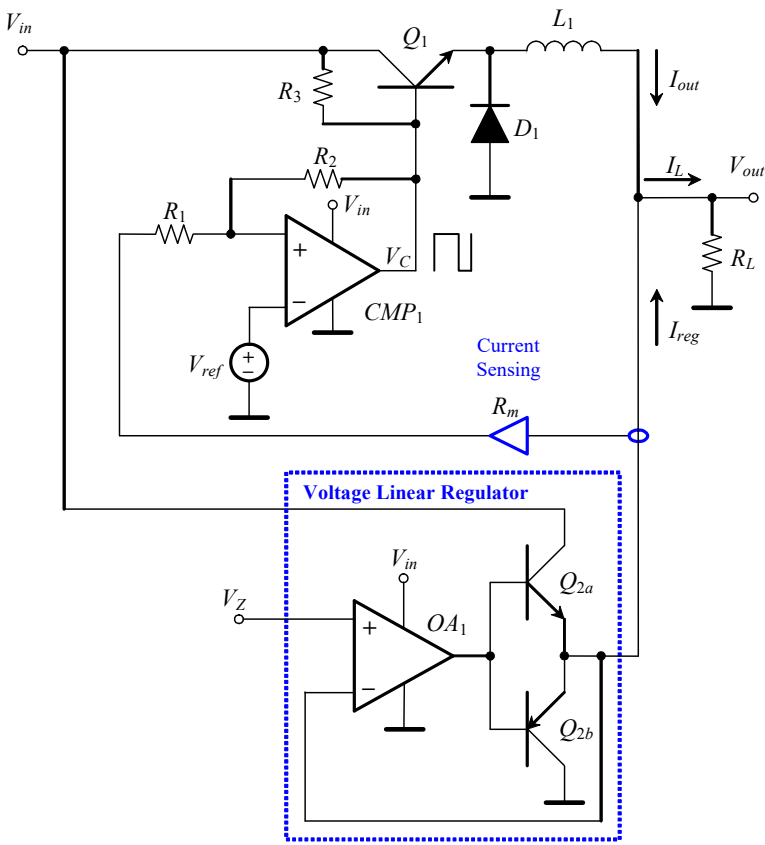

Fig. 4. Basic structure of linear-assisted converter with control strategy ' $B$ '.

As a matter of fact, if the current demanded by the load is inferior to a maximum value of current, which we will denominate switching threshold current, $\boldsymbol{I}_{\gamma}$, the output of comparator $C M P_{1}$ will be at low level, disabling the DC/DC switching converter and, thus, the current through inductor $L_{1}$ will be zero. Therefore, the voltage linear regulator supplies the load $R_{L}$, providing all the output current $\left(I_{\text {reg }}=I_{\text {out }}\right)$. 
When the current demanded by the load overpasses this current limit $\mathrm{I}_{\gamma}$, automatically the output of the comparator will pass to high level, causing that the current through the inductance $\mathrm{L}_{1}$ grows linearly according to:

$$
i_{L}(t)=\frac{V_{\text {in }}-V_{\text {out }}}{L_{1}} t+I_{L}\left(\tau_{1}\right)
$$

In that expression, the conduction collector-emitter voltage of transistor $Q_{1}$ is ignored. $I_{L}\left(\tau_{1}\right)$ is the initial value of the current through inductor $L_{1}$ at the time instant $T_{O N}$. Considering that the output current $I_{\text {out }}=I_{\text {reg }}+I_{L}$, and is assumed to be constant (equal to $V_{\text {out }} / R_{L}$ ), the linear regulator current $I_{\text {reg }}$ will decrease linearly, until becoming slightly smaller than $I_{\gamma}$. At this moment, the comparator will change its output to low level, cutting the transistor $Q_{1}$ and causing that the current through the inductor decreases according to equation (2):

$$
i_{L}(t)=-\frac{V_{o u t}}{L_{1}} t+I_{L}\left(\tau_{2}\right)
$$

In this expression, it is considered that the diode $\mathrm{D}_{1}$ is ideal (with zero direct voltage). $I_{L}\left(\tau_{2}\right)$ is the maximum value reached by the current flowing through the inductor (just at the beginning of the interval $T_{O F F}$ ). When the inductor current decreases to a value in which $I_{r e g}>I_{\gamma}$, the comparator changes its state to high level, repeating the cycle again.

Without hysteresis in the comparator, the switching point of the DC/DC switching converter is given by the switching threshold current, $I_{\gamma}$, of the linear regulator. This one can be adjusted to a value thanks to the gain of the current sensing element, $R_{m}$, and the reference voltage $V_{\text {ref }}$, according to the expression:

$$
R_{m}=\frac{V_{r e f}}{I_{\gamma}}
$$

In case of a comparator without hysteresis, intrinsic delays of the electronic circuits determine a small hysteresis that limits the maximum value of the linear-assisted converter switching frequency (figure 5). However, with the objective of fixing this switching frequency to a practical value, in order not to increase significantly losses by the switching process, it is important to add the aforementioned hysteresis to the comparator $C M P_{1}$.

Note that, in contrast to strategy ' $A$ ', with this new strategy, the linear regulator can be considered as the "main" block, and the switching converter as the auxiliary block. This one "assists" to the first one when the linear regulator supplies an excess current bigger than threshold $I_{\gamma}$.

It is important to emphasize that reducing the value of the power dissipated in the pass transistor of the linear regulator increases the efficiency of the set, even with significant output currents. Therefore, it is important to fix the current limit $I_{\gamma}$ to a commitment value between the minimum necessary to operate the regulator properly but without penalizing its good characteristics of regulation. What is more, with the purpose of obtaining a good regulation of the output, without significant ripple in it, it is mandatory that the current provided by the linear regulator is bigger than zero.

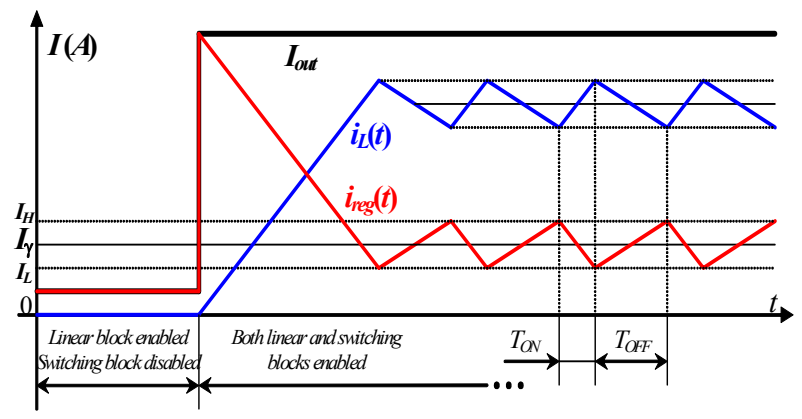

Fig. 5. Principle of operation of a linear-assisted converter with control strategy ' $B$ '.

The output voltage and the base-emitter voltage of transistors $Q_{2 a}$ and $Q_{2 b}$ at steady state are shown in figure 6. Comparing these graphs with those in figure 3 , shown in the same conditions, it becomes evident that with the proposed approach (realization ' $B$ ') the output ripple is reduced significantly.

In figure 7 we can appreciate the system behavior when the threshold current $I_{\gamma}$ is $5 \mathrm{~mA}$. Notice that the regulation of the output voltage gets worse significantly. It is accentuated when $I_{\gamma}$ is reduced down to a value near to zero. Therefore, the switching current threshold must be a value such that: (1) It significantly does not increase the power dissipation of the pass transistor in the linear regulator and does not diminish excessively the efficiency of the regulator, and (2) It does not significantly deteriorate the regulation of the output voltage.

Thus, we can denominate this type of control as a strategy control with nonnull average linear regulator current. For instance, for a practical implementation of a linearassisted converter using discrete components on board, for load currents lower than $10 \mathrm{~A}$, the suitable value of $I_{\gamma}$ that fulfills the two previous conditions is found to be between $10 \mathrm{~mA}$ and $50 \mathrm{~mA}$.

Finally, notice that if the load current is below $I_{\gamma}$, the switching block (the DC/DC converter) is disabled in order to minimize its losses. Thus, only the linear regulator provides the output current for slight load conditions. Figure 8 shows the experimental efficiency versus the load current for the two strategies. It is shown the comparison of the control strategy ' $A$ ' and the proposed by authors (strategy ' $B$ ') for $V_{\text {out }}=5 V$. It is shown four different $I_{\gamma}$ values: $0 m A$ (strategy ' $A$ '), and $10 m A, 50 m A$ and $100 \mathrm{~mA}$ (all three for strategy ' $B^{\prime}$ ). Note that, if $I_{\gamma}$ is low, the efficiency is not almost affected, reducing the output ripple too. 


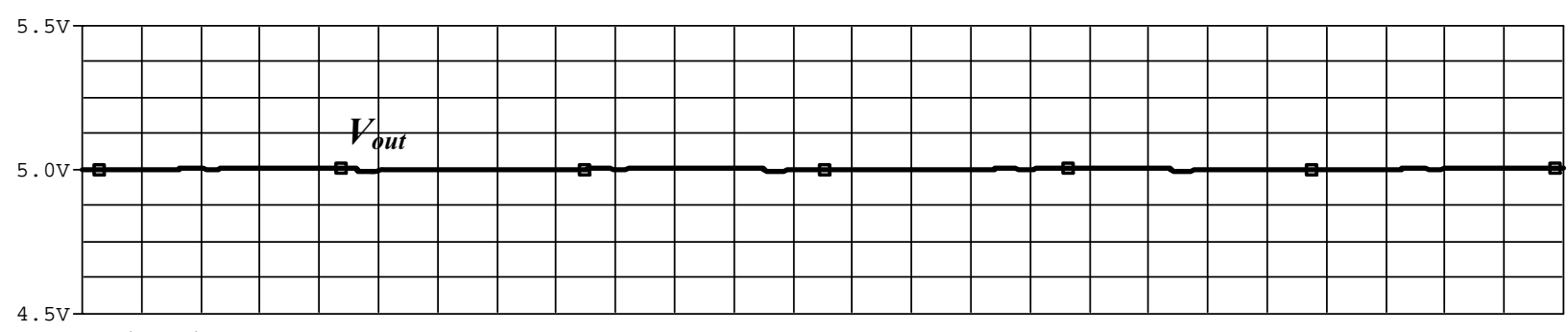

$4.5 \mathrm{~V} \frac{\mathrm{V}(\mathrm{Vout})}{\mathrm{a}}$

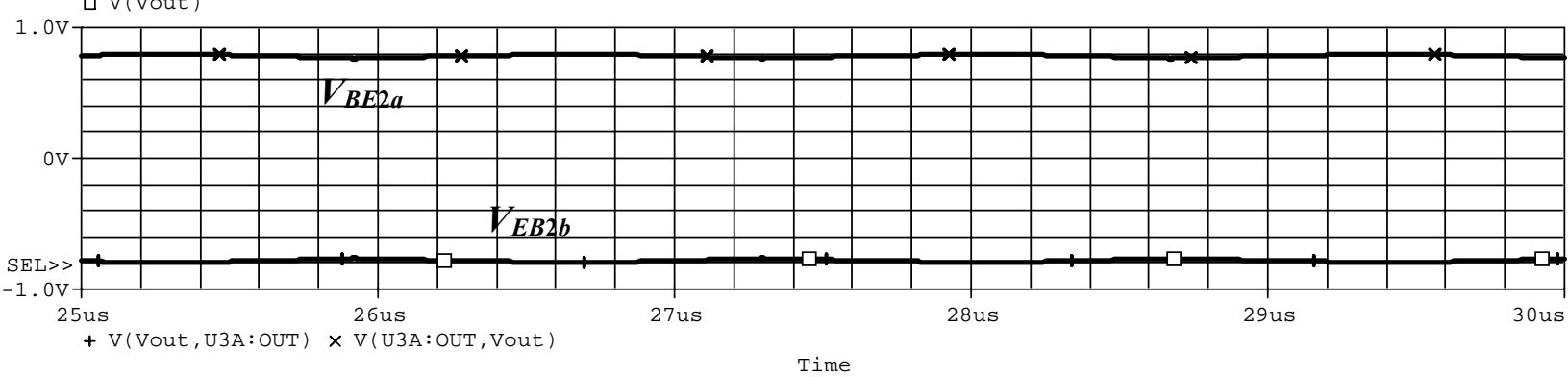

Fig. 6. Detail of the output voltage ripple and base-emitter voltage of transistors $Q_{2 a}$ and $Q_{2 b}$ of the linear regulator in the steady state in figure 4.

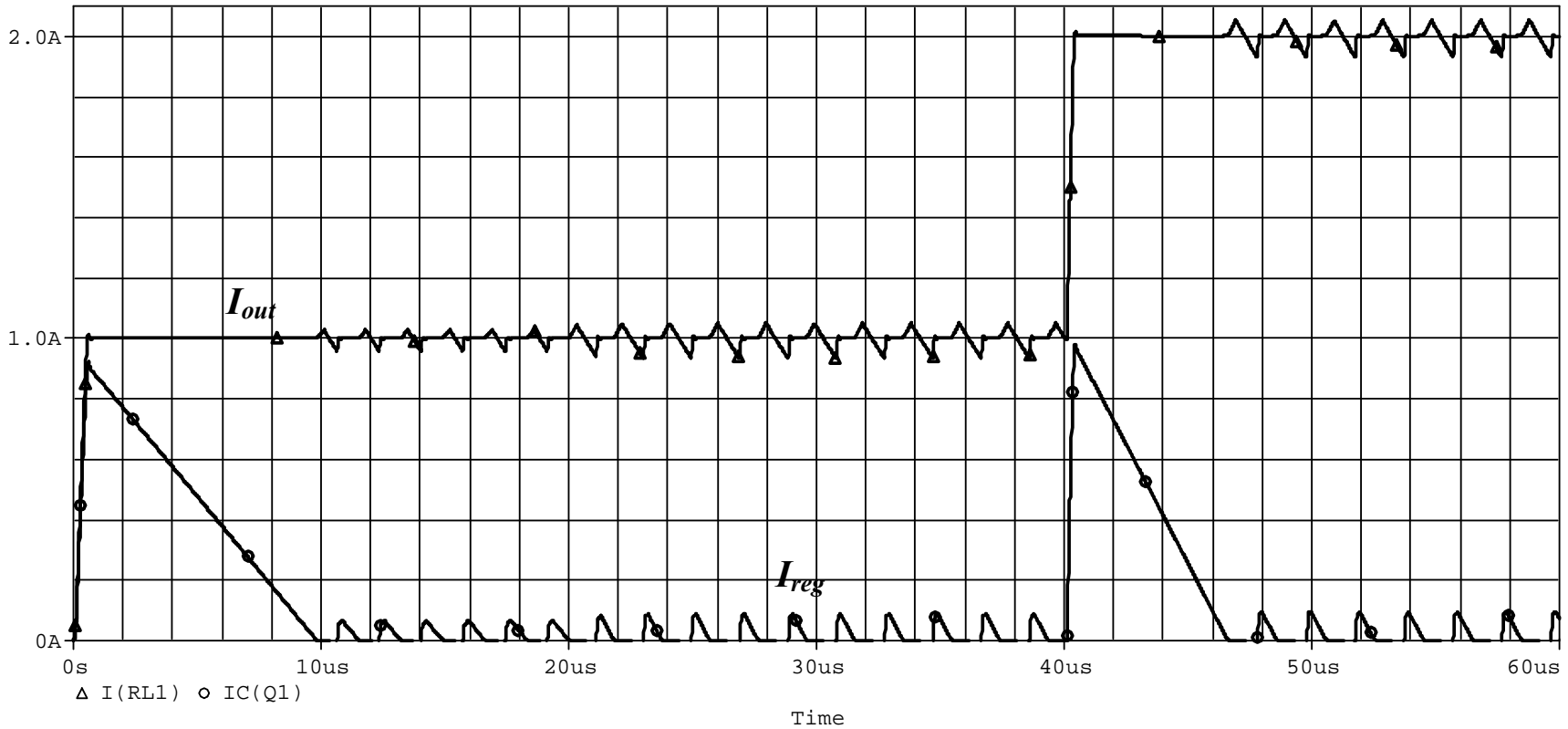

Fig. 7. Detail of the output current $I_{\text {out }}$ and the linear regulator current $I_{\text {reg }}$ at the start transient of the converter in figure 4 with $V_{i n}=10 \mathrm{~V}$. It can also be observed the response of the circuit to an input voltage step from $10 \mathrm{~V}$ to $13 V$ at $t=20 \mu \mathrm{s}$, and a variation in the load resistance from $5 \Omega$ a $2.5 \Omega$ at $t=40 \mu s$. The switching threshold current $I_{\gamma}$ is fixed at $5 \mathrm{~mA}$.

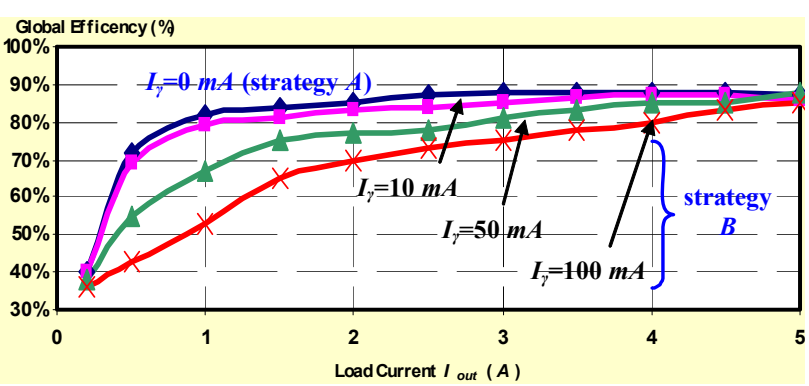

Fig. 8. Experimental efficiency for the control strategy ' $A$ ' and the proposed by the authors (strategy ' $B$ ') with $V_{\text {out }}=5 \mathrm{~V}$. It is shown four different $I_{\gamma}$ values: $0 \mathrm{~mA}$ (strategy $A$ ), and $10 \mathrm{~mA}, 50$ $m A$ and $100 m A$ (all three for strategy ' $B$ ').

\section{Conclusion}

This paper has shown the comparative of two strategies of control sensibly different for power DC/DC linear-assisted (or hybrid) converters based on the association of a linear regulator in parallel with a switching converter. The first of the two strategies (strategy ' $A$ ' or with null average value in the linear regulator current) allows to obtain a high efficiency, similar to switching converters because, in the steady state, the power dissipated in the linear regulator is practically zero. However, it has as inconvenient the presence of a ripple output voltage because the pass transistor of the output linear stage is switching between the cut and the conduction in every switching period. 
The proposal presented in the article (control strategy ' $B$ ' or nonnull average value in the linear regulator current), allows a little current through the linear stage that causes that the efficiency of the set diminishes slightly. However, it allows to obtain an output voltage practically free of spurious ripples.

This paper allows to affirm that, the maximum value of the current that circulates through the linear regulator (switching threshold current), must be fixed to a commitment value so that it does not increase the power dissipation in the pass transistor of the linear regulator significantly and does not make excessively diminish the efficiency of the set, but does not deteriorate significantly the regulation of the output voltage.

Finally, note, as an additional advantage of linear-assisted converters with this second strategy, that typical low pass filter capacitors, which are required in switching converters (and whose values, in certain applications, may become important), in this case they can be suppressed, since the linear regulator already makes the low pass filter function. Therefore, from this point of view, it can be said that, in an effective form, the voltage linear regulator acts as an active low pass filter, removing high frequency components generated in the modulation process.

\section{Acknowledgement}

This work has been partially supported by the Spanish Ministerio de Economía y Competitividad by project DPI2013-47799-C2-2-R

\section{References}

[1] R. Vázquez, A. Barrado, E. Olías, A. Lázaro. 'Theoretical Study and Implementation of a High Dynamic Performance, High Efficiency and Low Voltage Hybrid Power Supply'. Proceedings of the IEEE 32nd Annual Power Electronics Specialists Conference, 2001 (PESC 2001), vol. 3: pp. 1517-1522. 17-21 June 2001.

[2] A. Barrado, R. Vázquez, E. Olías, A. Lázaro, J. Pleite. 'Theoretical Study and Implementation of a Fast Transient Response Hybrid Power Supply'. IEEE Transactions on Power Electronics, vol. 19 (no 4): pp. 1003-1009, July 2004.

[3] H. Martínez, A. Conesa. "Modeling of Linear-Assisted DCDC Converters". European Conference on Circuit Theory and Design 2007 (ECCTD 2007), 26th-30th August 2007.

[4] A. Conesa, H. Martínez, J. M. Huerta. "Modeling of Linear \& Switching Hybrid DC-DC Converters". 12th European Conference on Power Electronics and Applications (EPE 2007), September 2007. 